\title{
Long non-coding RNA-DUXAP8 regulates TOP2A in the growth and metastasis of osteosarcoma via microRNA-635
}

\author{
TING YANG ${ }^{1}$, JIAN PING GUO ${ }^{2}$, FAN LI $^{2}, \mathrm{CHAO} \mathrm{XIU}^{3}, \mathrm{HUA} \mathrm{WANG}^{4}$ and XIAN LIANG DUAN ${ }^{2}$ \\ ${ }^{1}$ Department of Nephrology and Rheumatism Immunology, ${ }^{2}$ The Second Department of Orthopedics, ${ }^{3}$ Medical Imaging Center, \\ and ${ }^{4}$ The Department of Orthopedics, The Affiliated Hospital of Beihua University, Jilin, Jilin 132011, P.R. China
}

Received November 21, 2020; Accepted March 26, 2021

DOI: $10.3892 / \mathrm{mmr} .2021 .12150$

\begin{abstract}
Osteosarcoma (OS) is a malignant disease with high morbidity and mortality rates in children and adolescents. Evidence has indicated that long non-coding RNAs (lncRNAs) may serve important roles in human cancer progression, including OS. In the present study, the role of lnc-double homeobox A pseudogene 8 (DUXAP8) in the development of OS was identified. The expression of IncRNA-DUXAP8 was determined by reverse transcription-quantitative polymerase chain reaction in OS tissues. Cell proliferation was evaluated using Cell Counting kit-8 and colony formation assays, and Transwell assays were conducted to measure cell invasion. Cell migration was evaluated using a wound healing assay. The binding site between lnc-DUXAP8 and miR-635 RNAs was investigated using a luciferase reporter assay. The expression of lnc-DUXAP8 was significantly upregulated in OS samples and OS cell lines compared with normal tissues. High expression of lncRNA DUXAP8 was associated with shorter overall survival times. Knockdown of lncRNA DUXAP8 inhibited proliferation, migration and invasion in OS cells. Notably, mechanistic investigation revealed that IncRNA DUXAP8 predominantly acted as a competing endogenous RNA in OS by regulating the miR-635/topoisomerase alpha 2 (TOP2A) axis. IncRNA DUXAP8 is upregulated in OS, and lncRNA DUXAP8-knockdown serves a vital antitumor role in OS cell progression through the miR-635/TOP2A axis. The results of the present study suggested that lncRNA DUXAP8 may be a novel, promising biomarker for the diagnosis and prognosis of OS.
\end{abstract}

\section{Introduction}

Osteosarcoma (OS) is the most common primary sarcoma of the bone and mainly affects adolescents and children (1).

Correspondence to: Dr Xian Liang Duan, The Second Department of Orthopedics, The Affiliated Hospital of Beihua University, 12 Jie Fang Zhong Road, Jilin, Jilin 132011, P.R. China E-mail:714418649@qq.com

Key words: lncRNA DUXAP8, miR-635, TOP2A, osteosarcoma
Due to its high degree of malignancy, early metastasis, low chance of surgery, easy recurrence and high mortality, OS causes an unacceptable mortality rate (2). Although significant improvements have been made in the treatment of OS over the past decade, the prognosis of osteosarcoma remains poor (3). A previous study reported that the 5-year survival rate of OS patients without metastasis is $\sim 60-70 \%$ (4). However, the 5-year survival rate of OS patients with distant metastasis is only 20-30\% (5). Therefore, it is of paramount importance to investigate the molecular mechanisms underlying the pathogenesis of OS development.

Long non-coding RNAs (lncRNAs) are a type of non-coding nucleic acid with a length of $>200$ nucleotides and diverse and largely uncharacterized biological functions (6). Recently, increasing evidence has demonstrated that IncRNAs participate in fundamental cellular processes, including proliferation, migration and apoptotic processes, which are important in the development of cancer $(7,8)$. Previous studies have reported that lncRNAs function as oncogenes or tumor suppressors and are associated with cancer initiation and development, and lncRNAs may be dysregulated in various types of human cancer, including OS (9). For example, lncRNA SUMO1P3 promotes gastric cancer progression and invasion by regulating the EMT signaling pathway (10), and IncRNA AFAP1-AS1 accelerates nasopharyngeal carcinoma metastasis by sponging miR-423-5p to regulate the Rho/Rac pathway (11). These findings indicated that $\operatorname{lncRNAs}$ may be vital regulators during tumorigenesis and tumor progression.

In recent years, pseudogene-derived lncRNA double homeobox A pseudogene 8 (DUXAP8) has been shown to be upregulated in various malignant tumor types. Previous studies have reported that DUXAP8 works as an oncogene in renal cell carcinoma, gastric cancer and other tumor types $(12,13)$. A recent study reported that in HCC, DUXAP8 repressed tumor suppressor KLF2 transcription by interacting with EZH2 (14). However, the expression status and prognostic value of DUXAP8 in OS remain unknown.

MicroRNAs (miRNAs) are 22-nucleotide-long non-coding RNA molecules that can regulate target gene expression levels by binding to the 3'-untranslated regions (3'-UTRs) of target genes at the posttranscriptional level and promoting degradation or inhibiting translation (15). 
miR-635 is located in $17 \mathrm{q}$ and has been recently identified in colorectal cancer (16). Weber et al (17) reported that miR-635 may significantly accelerate the invasion of A375 melanoma cells. However, the mechanism of miR-635 regulation in OS requires further investigation. Topoisomerase alpha 2 (TOP2A) is a marker of proliferation and chemotherapy resistance in different cancer types, including adrenocortical carcinoma and breast carcinoma $(18,19)$. Furthermore, it has been reported that several miRNAs serve a regulatory role by directly inhibiting the target TOP2A in cancer (20).

In the present study, it was demonstrated for the first time that DUXAP8 was enhanced in OS cell lines and tissues. Downregulation of DUXAP8 markedly suppressed OS cell viability and invasion. Additionally, it was confirmed that DUXAP8 may promote the development of OS cells by modulating miR-635/TOP2A. The results of the present study may offer a novel diagnostic and therapeutic candidate for OS treatment.

\section{Materials and methods}

Patient samples. Patients with OS $(n=35)$ who received surgery in the Affiliated Hospital of Bei Hua University (Jilin, China) between October 2018 and October 2019 were selected to obtain cancer tissue samples and adjacent normal tissues. The patients were 31-73 years old, including 19 males and 16 females and they had not received chemotherapy or radiotherapy prior to surgery. The tissues were subsequently stored in liquid nitrogen and then stored at $-80^{\circ} \mathrm{C}$ until extraction of RNA. All research protocols in the present study were approved by the Ethics Committee of the Affiliated Hospital of Bei Hua University. Written informed consent was obtained from every patient.

Cell lines and cell culture. Human osteosarcoma cell lines, including KHOS-240S, SaOS2, MG-63, SOSP-9607 and U2OS, and one normal osteoblastic cell line (hFOB1.19) were obtained from the American Type Culture Collection and the Cell Bank of the Chinese Academy of Sciences, respectively. All cell lines were cultured according to the manufacturer's protocols. Cells were cultured in Dulbecco's modified Eagle's medium, supplemented with $10 \%$ fetal bovine serum, and all incubations were performed at $37^{\circ} \mathrm{C}$ in a $5 \% \mathrm{CO}_{2}$-containing atmosphere.

Cell transfection. The short interfering RNAs that targeted lncRNA DUXAP8 (si-lncRNA-DUXAP8), corresponding siRNA negative controls (siNC), miR-635 mimic, negative control (NC) miRNA, miR-635 inhibitor and NC inhibitor were purchased from Shanghai GenePharma Co., Ltd. Transfections were performed using the Lipofectamine 3000 kit (Invitrogen; Thermo Fisher Scientific, Inc.), according to the manufacturer's protocol. RNAs $(100 \mathrm{nM})$ or miR-635 mimics $(50 \mathrm{nM})$ or miR-635 inhibitor $(150 \mathrm{nM})$ or plasmids (1.5 $\mu \mathrm{g}$ per well) were transfected into cells. The sequences were as follows: si-DUXAP8, 5'-AAGAUAAAGGUGGUU UCCACAAGAATT-3'; si-NC, 5'-AGCUUGAUACGACAA AGCUTT-3'; miR-635 mimic, 5'-ACUUGGGCACUGAAA CAAUGUCC-3'; miR-NC, 5'-CAGUACUUUUGUGUA GUACAA-3'; miR-635 inhibitor, 5'-GGACAUUGUUUCAGU
GCCCAAGU-3'; and inhibitor NC, 5'-CAGUACUUUUGU GUAGUACAA-3'.

Transfection was performed at room temperature for $30 \mathrm{~min}$. The knockdown efficiency was assessed by reverse transcription-quantitative (RT-q) PCR $48 \mathrm{~h}$ after transfection, when the cells were collected for the subsequent experiments.

$R N A$ extraction and $R T-q P C R$. Total RNA was extracted from tissues and cells using TRIzol (Invitrogen; Thermo Fisher Scientific, Inc.), according to the manufacturer's protocol. Total RNA was reverse transcribed into cDNA using the Prime Script ${ }^{\circledR}$ RT Reagent kit. RT reaction was conducted for $15 \mathrm{~min}$ at $42^{\circ} \mathrm{C}$ followed by $5 \mathrm{~min}$ at $98^{\circ} \mathrm{C}$ and the reaction volume was $20 \mu \mathrm{l}$. qPCR was performed using SYBR Premix Ex Taq (Takara Biotechnology Co., Ltd.) on an ABI 7500 RT-qPCR system. Expression of DUXAP8 and TOP2A was detected using GAPDH as endogenous control, respectively. High Pure miRNA Isolation kit (Sigma-Aldrich; Merck KGaA) was used to extract miRNA. miRNA reverse transcription was performed using MystiCq ${ }^{\circledR}$ microRNA cDNA Synthesis mix (Sigma-Aldrich; Merck KGaA), and qPCR was performed using MystiCq microRNA ${ }^{\circledR}$ SYBR $^{\circledR}$ Green qPCR ReadyMix ${ }^{\circledR}$ (Sigma-Aldrich; Merck KGaA) to measure the level of miR-635 expression with U6 as an endogenous control. The primer sequences were as follows: DUXAP8 forward, 5'-AGG ATGGAGTCTCGCTGTATTGC-3' and reverse, 5'-GGAGGT TTGTTTTCTTCTTTTTT-3'; TOP2A forward, 5'-GATTGA TTATGACAAAGTATA-3' and reverse, 5'-TACTTTGTC ATAATCAATCAG-3'; GAPDH forward, 5'-CGCTCTCTG CTCCTCCTGTTC-3' and reverse, 5'-ATCCGTTGACTC CGACCTTCAC-3'. miR-635 forward, 50-TATAGCATATGC AGGGTG-30; miR-635 reverse primer and U6 primers were included in the kit. The thermocycling conditions were as follows: Initial denaturation at $95^{\circ} \mathrm{C}$ for $10 \mathrm{~min}$, followed by 40 cycles at $95^{\circ} \mathrm{C}$ for $15 \mathrm{sec}$ and extension at $60^{\circ} \mathrm{C}$ for $1 \mathrm{~min}$. The relative expression of DUXAP8, miR-635 and TOP2A mRNA levels was calculated using the $2^{-\Delta \Delta \mathrm{Ct}}$ method (21). The median value was the cut-off between low and high DUXAP8 expression in patients with OS. The median value was included in the low expression group.

Cell proliferation assay. Cell proliferation was quantified using Cell Counting Kit-8 (CCK-8; Beyotime Institute of Biotechnology), according to the manufacturer's protocols. In brief, $1 \times 10^{5} /$ well cells were seeded and transfected into a 96-well plate (Corning Incorporated). At the indicated times, $10 \mu \mathrm{l}$ CCK-8 solution was added to each well, and the cells were incubated for $4 \mathrm{~h}$ at $37^{\circ} \mathrm{C}$. The absorbance was measured using a microplate reader (BioTek Instruments, Inc.) at $450 \mathrm{~nm}$.

Wound healing assay. To measure the migratory ability of OS cells, a wound-healing assay was performed. Cells were seeded and cultured to a confluent monolayer in a rectangular cell culture plate. The medium was removed, and then the teeth of the cell comb were drawn across the cell monolayer with sufficient force. Cells were washed, replenished with fresh Dulbecco's modified Eagle's medium (DMEM; Thermo Fisher Scientific, Inc.) and incubated for an additional $24 \mathrm{~h}$. Wound closure was monitored with a light microscope (magnification, x100; BX51; Olympus Corporation). Gap distance was 
quantified using NIH ImageJ software version 1.46 (National Institutes of Health).

Transwell assay. Transwell membranes with $8-\mu \mathrm{m}$ pore sizes (Corning Incorporated) coated with Matrigel (BD Biosciences) were used for the cancer cell invasion assay as previously described (22). Following the indicated transfection, $2 \times 10^{5}$ cells were resuspended in fresh serum-free DMEM and replated into the upper chamber. Fresh DMEM containing 10\% fetal bovine serum (FBS) was directly added to the lower chamber. After an additional $24 \mathrm{~h}$ of incubation at $37^{\circ} \mathrm{C}$, the invasive cells penetrated the lower surface, were fixed with $4 \%$ paraformaldehyde in phosphate-buffered saline (PBS) at room temperature for $10 \mathrm{~min}$ and stained with $0.1 \%$ crystal violet at room temperature for $10 \mathrm{~min}$. (Sigma-Aldrich; Merck KGaA). The number of invasive cells was counted under a light microscope (magnification, x100; BX51; Olympus Corporation).

Colony formation assay. A colony formation assay was also performed. Cells were added to 6 -well plates $\left(2 \times 10^{3}\right.$ cells/well $)$ following transfection for 2 weeks. Colonies were fixed with $100 \%$ methanol at room temperature for $20 \mathrm{~min}$ and stained with $0.1 \%$ crystal violet (Sigma-Aldrich; Merck $\mathrm{KGaA}$ ) at $25^{\circ} \mathrm{C}$ for $30 \mathrm{~min}$. The total number of visible colonies was imaged and counted using a light microscope (magnification, x100). All experiments were repeated three times.

Luciferase activity assay. The wild-type (WT) or mutant (MUT) DUXAP8 sequences containing the miR-635 binding sites were cloned into the pmir-GLO Dual-luciferase vector (Promega Corporation) and co-transfected with the miR-635 mimics or corresponding control sequences into the cells with Lipofectamine $^{\circledR} 2000$ (Invitrogen; Thermo Fisher Scientific, Inc.). The sequences were WT DUXAP8: 5'-UUUAAAACU CUUGAUGCUGGUU-3'; MUT DUXAP8: 5'-UUUAUU UGAGUUGAUGCUGGUU-3' miR-635 mimic, 5'-ACUUGG GCACUGAAACAAUGUCC-3'; miR-NC, 5'-CAGUACUUU UGUGUAGUACAA-3'. Following transfection, the cells were incubated for $48 \mathrm{~h}$ and the luciferase activity was measured with the Dual Luciferase Reporter Assay System (Promega Corporation). The Renilla luciferase was used as an internal control to homogenize the detection of the reporter gene.

Western blotting. Western blotting was performed following the protocol as previously described (23). In brief, cells were lysed with RIPA lysis buffer (Thermo Fisher Scientific, Inc.), incubated on ice for $30 \mathrm{~min}$, and centrifuged at 11,000 $\mathrm{x}$ g for $30 \mathrm{~min}$ at $4^{\circ} \mathrm{C}$. The supernatant was then collected, and the protein concentration was determined using a BCA protein quantitation kit (Beyotime Institute of Biotechnology). Cell lysates $(20 \mu \mathrm{g})$ were subjected to $10 \%$ SDS-PAGE and transferred to polyvinylidene fluoride membranes (GE Healthcare). Next, the membranes were blocked with $5 \%$ bovine serum albumin in PBS for $1 \mathrm{~h}$ at room temperature and incubated with primary antibodies TOP2A (cat. no. 12286, Cell Signaling Technology, Inc.; dilution, 1:1,000) and GAPDH (cat. no. 5174, Cell Signaling Technology, Inc.; dilution, 1:5,000) overnight at $4^{\circ} \mathrm{C}$. Next, the blots were washed and probed with a secondary antibody of HRP Goat Anti-Rabbit (IgG; cat. no. ab6721, Abcam; dilution, 1:10,000) at room temperature for $1.5 \mathrm{~h}$ and visualized using an ECL detection system (Thermo Fisher Scientific, Inc.). The relative densities of the protein bands were analyzed with NIH ImageJ software version 1.46 (National Institutes of Health).

Statistical analysis. Statistical analyses were performed using GraphPad Prism software (version 6; GraphPad Software, Inc.). A normal distribution and homogeneity of variance were tested. Measurement data conforming to a normal distribution were expressed as the mean \pm standard deviation. If the data did not conform to a normal distribution or homogeneity of variance, quantile spacing was applied. The sample size of each group for the cell experiments was nine. The relationship between lncRNA DUXAP8 and miR-635 expression levels in OS and normal adjacent tissues was analyzed by paired Student's t-test. Survival curves were plotted using the Kaplan-Meier method and log-rank tests were performed. An unpaired t-test was applied for the other comparisons between two groups. For the comparison of multiple groups, one-way ANOVA analysis was performed followed by Tukey's post hoc test. Correlations were analyzed using Pearson's correlation. Linear regression analysis was performed to identify variables that significantly affected these correlations. $\mathrm{P}<0.05$ was considered to indicate a statistically significant difference.

\section{Results}

The expression of IncRNA DUXAP8 is upregulated in OS tissues and cell lines. To investigate the role of lncRNA DUXAP8 in OS, the relative expression level of lncRNA DUXAP8 was investigated in 35 pairs of OS tissues and adjacent non-tumor tissues by RT-qPCR analysis. Differences in expression level of DUXAP8 between OS and non-tumor tissues were analyzed by paired t-test. As presented in Fig. 1A $(\mathrm{P}<0.001)$, the level of lncRNA DUXAP8 was higher in OS tissues than in non-cancerous samples. Additionally, the expression of DUXAP8 was investigated in 5 human OS cell lines (KHOS, SOSP-9607, U2OS, MG-63 and SaOS-2) and the normal osteoblastic hFOB1.19 cell line by RT-qPCR. The results revealed that lncRNA DUXAP8 expression was markedly increased in the five OS cell lines compared with the hFOB1.19 cell line (Fig. 1B; P<0.001). To further determine the association between DUXAP8 expression and the long-term prognosis of patients, Kaplan-Meier analysis was performed based on TCGA patients using GEPIA. Patients with higher DUXAP8 expression levels had shorter overall survival times than patients with lower DUXAP8 expression levels (Fig. 1C). These results suggested that DUXAP8 was involved in the progression of OS.

DUXAP8 promotes the proliferation, migration and invasion of OS cells. To determine the potential biological role of lncRNA DUXAP8 in OS cells, two OS cell lines, U2OS and SaOS2 cells, with higher expression of lncRNA DUXAP8 were selected to assess the effects of siRNA-mediated knockdown of lncRNA DUXAP8 on cell proliferation and colony formation. Following transfection with lncRNA DUXAP8-specific siRNAs or a control siRNA, IncRNA DUXAP8 expression was revealed to be efficiently decreased by RT-qPCR analysis (Fig. 2A; P<0.01). It was observed 

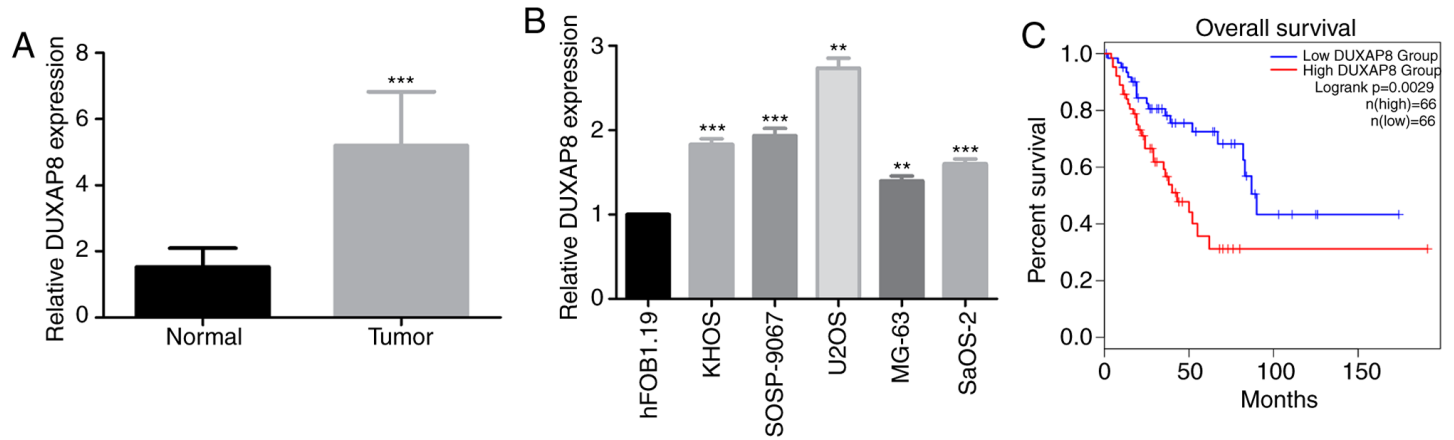

Figure 1. IncRNA DUXAP8 expression is increased in OS tissues and cell lines. (A) Expression of lncRNA DUXAP8 was measured using RT-qPCR in OS tissues $(n=35)$ and healthy adjacent tissues $(n=35)$. Data are expressed as the mean \pm standard deviation using Student's $t$-test. ${ }^{* * *} P<0.001$ vs. normal tissues. (B) RT-qPCR analysis was used to determine lncRNA DUXAP8 expression in KHOS, SOSP-9607, U2OS, MG-63 and SaOS-2 cells, as well as the normal osteoblastic hFOB1.19 cell line. ${ }^{* *} \mathrm{P}<0.01,{ }^{* * *} \mathrm{P}<0.001$ vs. hFOB1.19. (C) Survival analysis of OS patients with high and low expression of DUXAP8. Data were compared using the paired t-test. All PCR reactions were repeated 3 times and mean values are presented. IncRNA, long non-coding RNA; DUXAP8, double homeobox A pseudogene 8; OS, osteosarcoma; RT-qPCR, reverse transcription-quantitative polymerase chain reaction.

A

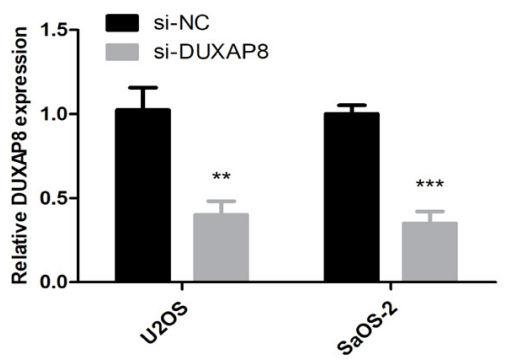

D
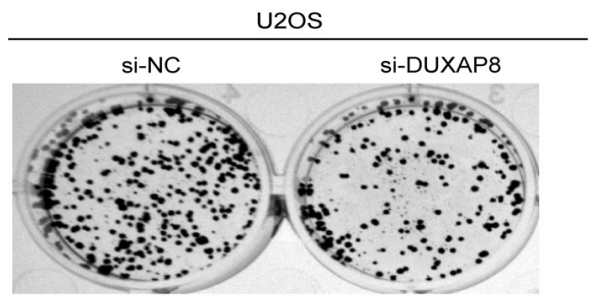

E
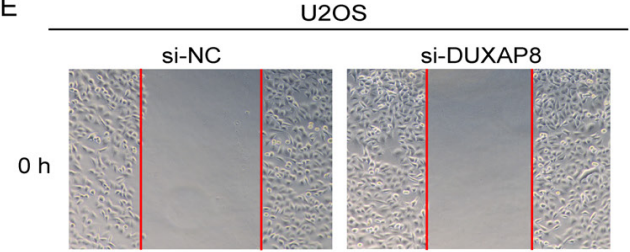

$48 \mathrm{~h}$

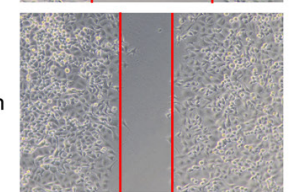

F

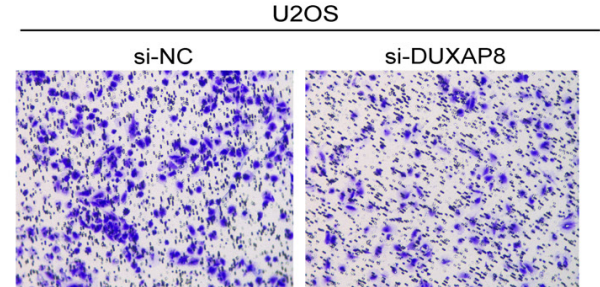

B

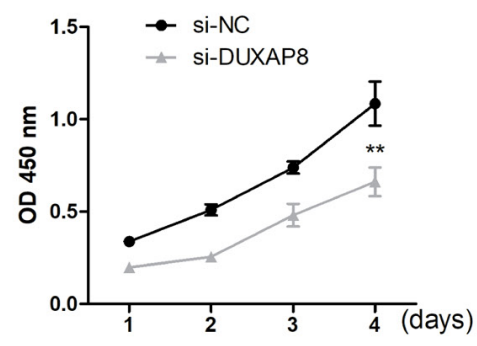

SaOS-2
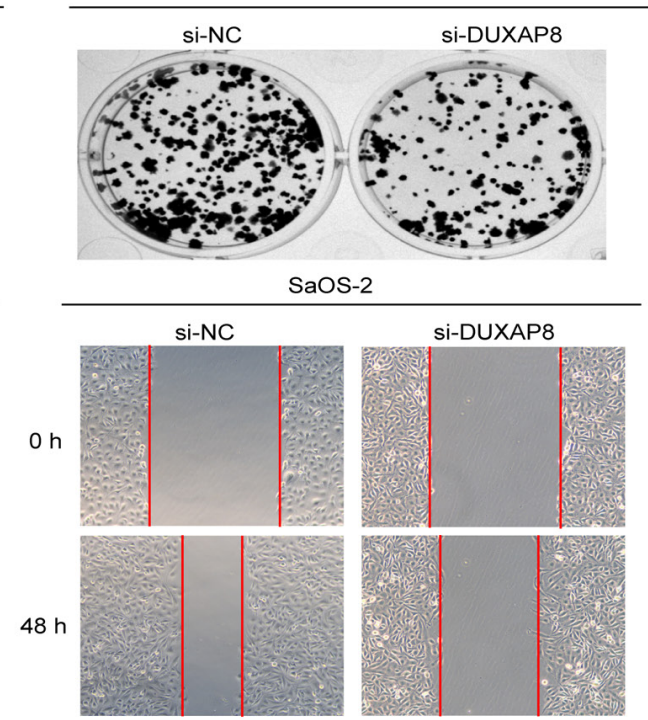

SaOS-2

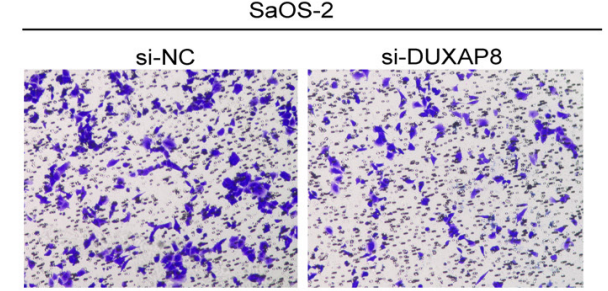

C

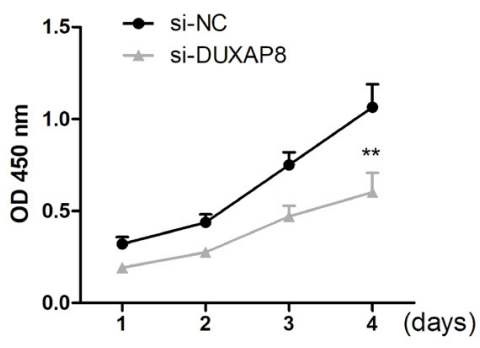

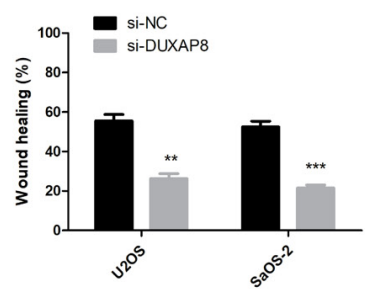

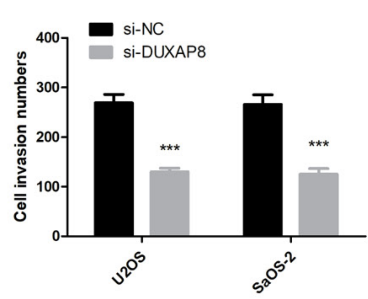

Figure 2. DUXAP8 promotes OS proliferation, migration and invasion. (A) lncRNA DUXAP8 expression was detected in U2OS and SaOS-2 cells transduced with a lncRNA DUXAP8 siRNA vector (si-lncRNA DUXAP8) or negative control siRNA vector (si-NC) ${ }^{* *} \mathrm{P}<0.01,{ }^{* * *} \mathrm{P}<0.001$ vs. si-DUXAP8. (B-D) Cell viability was measured using a Cell Counting kit- 8 assay and colony formation assay at the indicated times following transfection, ${ }^{* *} \mathrm{P}<0.01,{ }^{* * *} \mathrm{P}<0.001 \mathrm{vs}$. si-DUXAP8. (E) The migration of OS cells following DUXAP8-knockdown was detected by a scratch healing assay, ${ }^{* *} \mathrm{P}<0.01,{ }^{* * *} \mathrm{P}<0.001$ vs. si-DUXAP8 Magnification, $x 100$. (F) The invasion of OS cells following DUXAP8-knockdown was detected by a Transwell assay, ${ }^{* * *}$ P $<0.001$ vs. si-DUXAP8. Magnification, x100. Data are expressed as the mean \pm standard deviation, Student's t-test. DUXAP8, double homeobox A pseudogene 8; OS, osteosarcoma; lncRNA, long non-coding RNA; si, small interfering RNA; NC, negative control; OD, optical density. 
A

WT DUXAP8: 5'-UUUAAAACUCUUGAUGCUGGUU-3'

has-miR-635: 3'-CAGGUUUGAGCCCCACGACCAA-5'

MUT DUXAP8: 5'-UUUAUUUGAGUUGAUGCUGGUU-3'

D

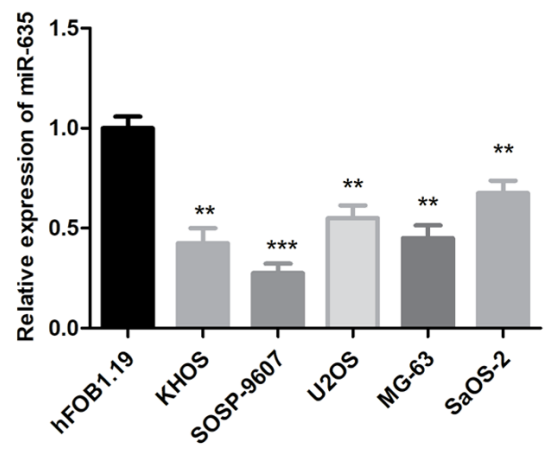

G

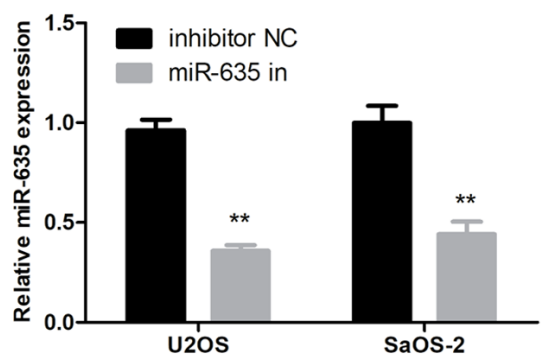

B

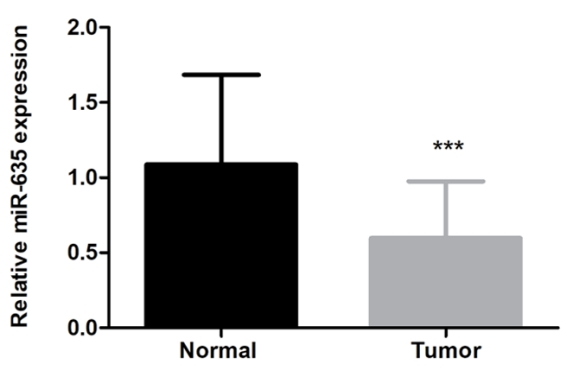

E

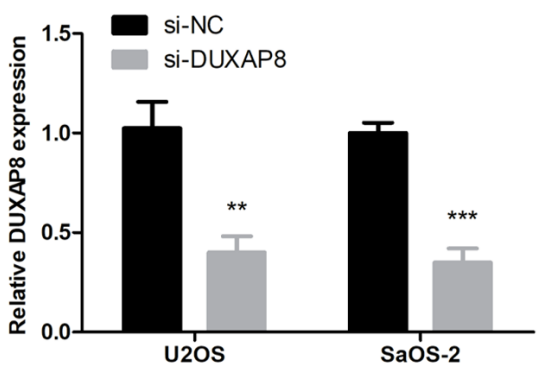

$\mathrm{H}$

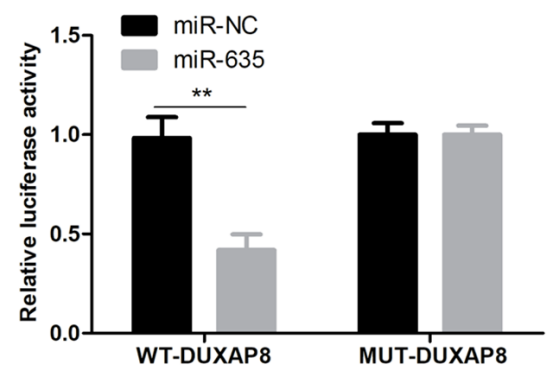

C

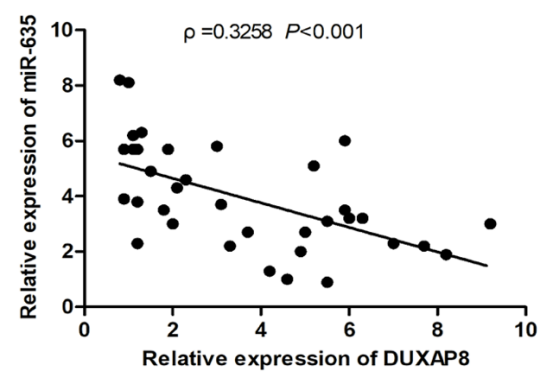

F
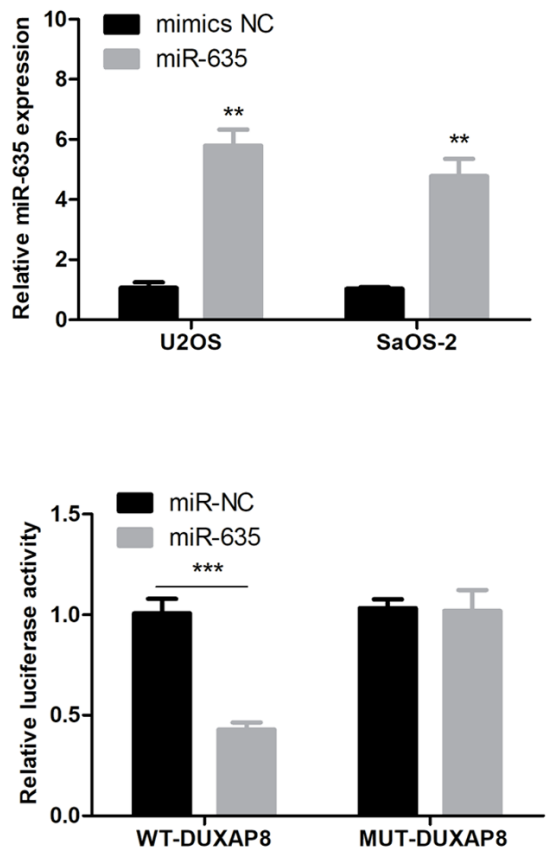

Figure 3. DUXAP8 targets miR-635 in OS. (A) Prediction of binding sites between miR-635 and DUXAP8. (B) The expression levels of miR-635 in 35 matched OS and adjacent non-tumor controls were detected by RT-qPCR, ${ }^{* * *} \mathrm{P}<0.001$ vs. normal tissues. (C) The correlation between the expression levels of DUXAP8 and miR-635 in OS samples was analyzed $\left({ }^{* * *} \mathrm{P}<0.001\right)$. (D) The expression of miR-635 in OS cell lines and the normal osteoblastic hFOB1.19 cell line was detected by RT-qPCR, ${ }^{* *} \mathrm{P}<0.01$ or ${ }^{* * * *} \mathrm{P}<0.001$ vs. hFOB1.19. (E) The expression levels of miR-635 in OS cell lines were detected by RT-qPCR following DUXAP8-knockdown, ${ }^{* *} \mathrm{P}<0.01,{ }^{* * *} \mathrm{P}<0.001$ vs. si-DUXAP8. (F) The expression levels of U2OS and SaOS-2 cells transfected with miR-635 or mimic negative control, ${ }^{* *} \mathrm{P}<0.01$ vs. miR-635. (G) The expression levels of U2OS and SaOS-2 cells transfected with NC inhibitor/miR-635 inhibitor, ${ }^{* *} \mathrm{P}<0.01$ vs. miR-635 in. (H and I) Dual-luciferase reporter assay indicated that miR-635 mimics could decrease the luciferase activity of the wild-type DUXAP8 reporter in U2OS and SaOS-2 cells, ${ }^{* *} \mathrm{P}<0.01,{ }^{* * *} \mathrm{P}<0.001$ vs. miR-635. DUXAP8, double homeobox A pseudogene 8; miR, microRNA; OS, osteosarcoma; RT-qPCR, reverse transcription-quantitative polymerase chain reaction; NC, negative control; si, small interfering RNA; WT, wild-type.

that knockdown of lncRNA DUXAP8 caused a significant decrease in cell proliferation, as measured using CCK-8 assays and colony formation assays (Fig. 2B-D; $\mathrm{P}<0.01$ ). The migration ability of U2OS and SaOS2 cells were further examined by scratch assay. The results demonstrated that the migration ability of U2OS and SaOS2 cells was significantly decreased following knockdown of DUXAP8 (Fig. 2E; $\mathrm{P}<0.001)$. The effects of DUXAP8 on the invasion of OS cells were investigated by Transwell assay. Furthermore, the results demonstrated that knockdown of DUXAP8 inhibited the invasion of U2OS and SaOS2 cells (Fig. 2F; P<0.001). Taken together, these results confirmed that DUXAP8 was an oncogenic lncRNA in OS.

DUXAP8 targets miR-635 in OS cells. 1ncRNAs may act as molecular sponges to regulate the expression of downstream genes by absorbing miRNAs. By searching for the potential binding miRNA of DUXAP8 in the StarBase online database (www.starbase.sysu. edu.cn), miR-635 was selected as a predictive target for DUXAP8 because of its high binding potential (Fig. 3A). The expression of miR-635 was significantly downregulated in OS tissues (Fig. 3B; P<0.001). Notably, the expression correlation between DUXAP8 and miR-635 was analyzed in OS tissue samples, and their expression was revealed to be negatively correlated (Fig. 3C; $\mathrm{P}<0.001$ ). The expression of miR-635 was significantly downregulated in OS cells (Fig. 3D; $\mathrm{P}<0.01)$. Additionally, an increase in miR-635 expression was observed in U2OS and SaOS2 cells with DUXAP8-knockdown, suggesting that DUXAP8 may negatively regulate miR-635 expression in OS (Fig. 3E; $\mathrm{P}<0.01$ ). U2OS and SaOS2 cells were transfected with miR-635 or negative control and the efficiency of transfection was determined by RT-qPCR. Results demonstrated that the ectopic transfection significantly upregulated miR-635 expression levels (Fig. 3F; P<0.001). Additionally, 
A

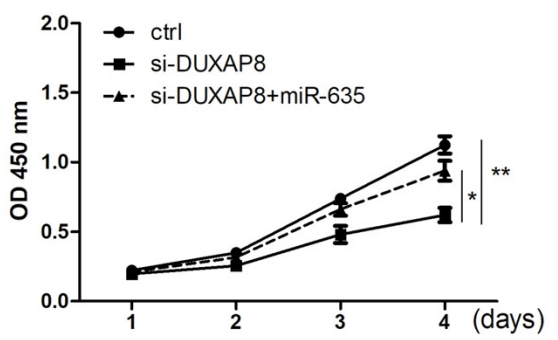

C
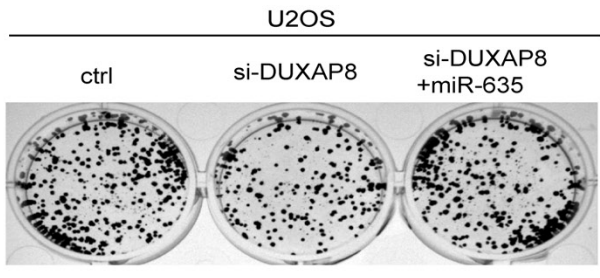

D
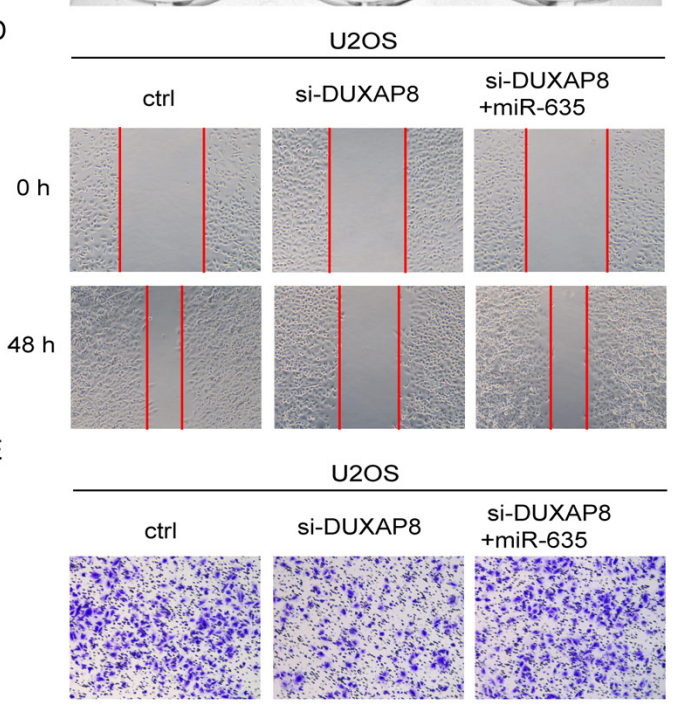

B
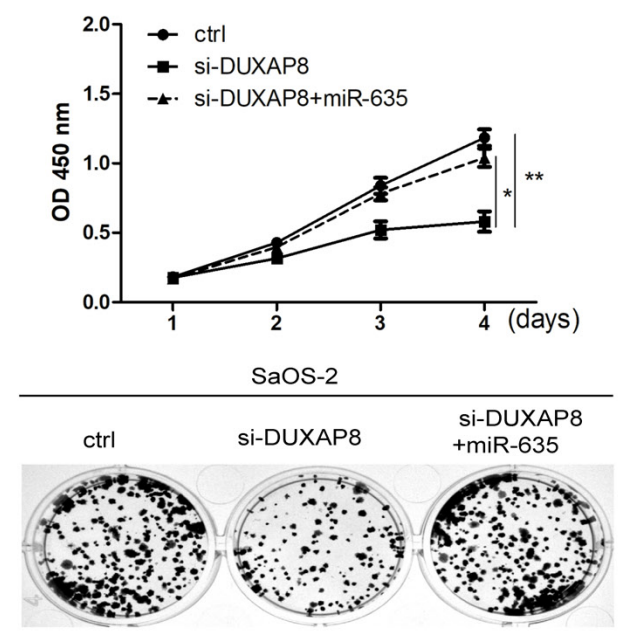

SaOS-2
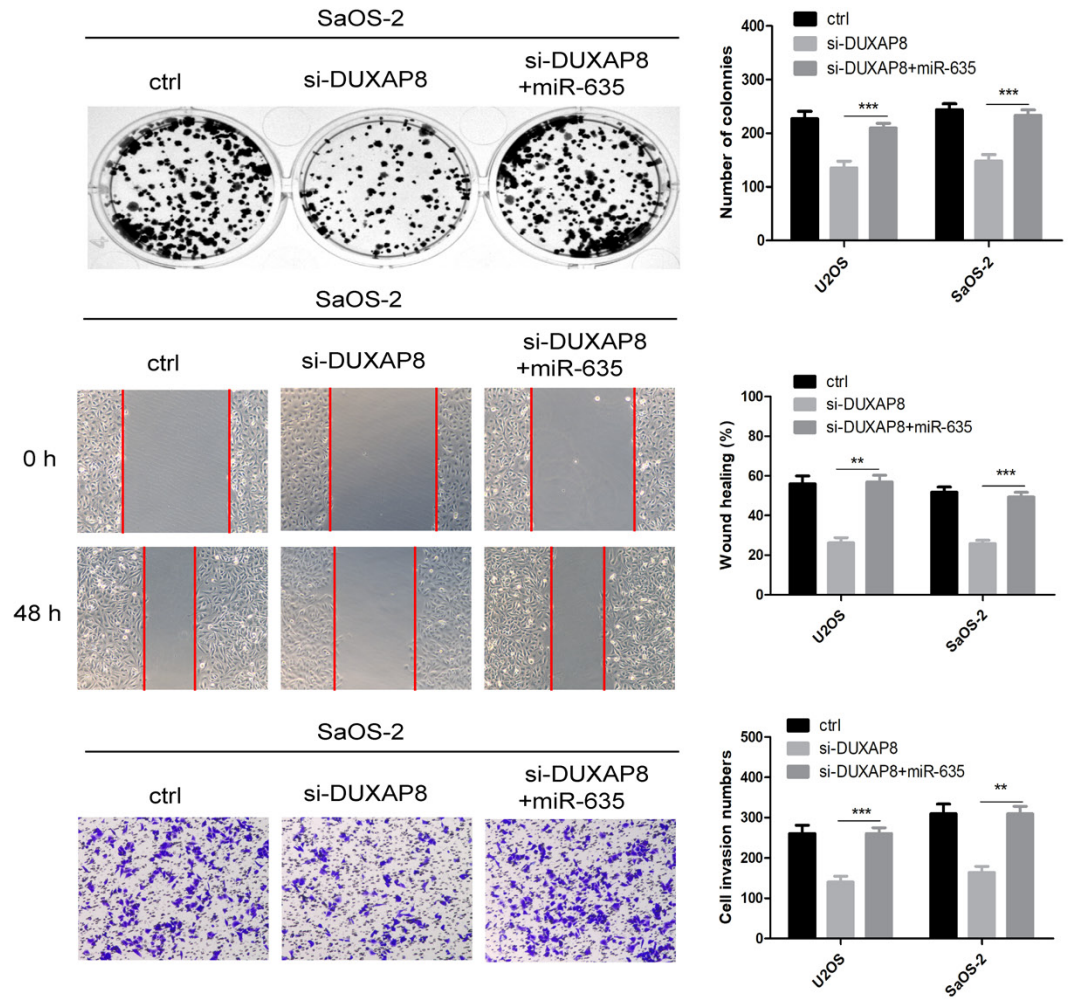

Figure 4. miR-635 reverses the function of DUXAP8 in OS. (A and B) miR-635 mimics and DUXAP8 siRNA were co-transfected into OS cells, and the proliferation of OS cells in each group was detected by Cell Counting kit- 8 assay, ${ }^{*} \mathrm{P}<0.05$ vs. si DUXAP8+miR-635, ${ }^{* *} \mathrm{P}<0.01$ vs. control. (C) The cell viability in each group was measured using a colony formation assay, ${ }^{* *} \mathrm{P}<0.01,{ }^{* * *} \mathrm{P}<0.001$ vs. si-DUXAP8. (D) The migration in each group was evaluated by scratch healing assay, ${ }^{* *} \mathrm{P}<0.01,{ }^{* * *} \mathrm{P}<0.001$ vs. si-DUXAP8. Magnification, $\mathrm{x} 100$. (E) The invasion of OS cells in each group was evaluated by Transwell assay, ${ }^{* *} \mathrm{P}<0.01,{ }^{* * *} \mathrm{P}<0.001$ vs. si-DUXAP8. Magnification, $\mathrm{x} 100$. Data are expressed as the mean \pm standard deviation, Student's t-test. miR, microRNA; DUXAP8, double homeobox A pseudogene 8; OS, osteosarcoma; siRNA, small interfering RNA; Ctrl, control; OD, optical density.

U2OS and SaOS2 cells were transfected with NC inhibitor/ miR-635 inhibitor and the efficiency of transfection was determined by RT-qPCR. Results demonstrated that the ectopic transfection significantly downregulated miR-635 expression levels (Fig. 3G; $\mathrm{P}<0.01$ ). To further validate the binding relationship between miR-635 and DUXAP8, a dual-luciferase reporter assay was performed and demonstrated that luciferase activity decreased significantly following U2OS or SaOS2 cells being co-transfected with miR-635 and DUXAP8-WT reporter plasmids; however, the luciferase activity did not decrease significantly when the cells were co-transfected with miR-635 and DUXAP8-MUT reporter plasmids (Fig. 3H and I; P<0.01). Taken together, these results indicated that DUXAP8 negatively regulated miR-635 in OS.

miR-635 may reverse the function of DUXAP8 in OS cells. Next, miR-635 mimics were transfected into U2OS or SaOS2 OS cells with DUXAP8-knockdown. It was revealed that overexpression of miR-635 attenuated the effect of overexpressing DUXAP8 on proliferation by CCK- 8 assays and colony formation assays (Fig. 4A-C, P<0.05). Next, scratch and Transwell assays were conducted. Overexpression of miR-635 attenuated the effect of overexpressing DUXAP8 on the migration and invasion of OS cells (Fig. 4D and E, P<0.01). These findings indicated that miR-635 reversed the function of DUXAP8 in OS cells.

Regulation of DUXAP8/miR-635 on TOP2A expression in OS cells. After confirming that DUXAP8 can regulate miR-635 expression, the downstream targets of miR-635 in OS were investigated. A recent study (24) demonstrated that TOP2A, an oncogene, is a target of miR-635; therefore, the present study investigated whether DUXAP8 can regulate TOP2A expression in OS. DUXAP8-knockdown significantly decreased the mRNA and protein expression of TOP2A (Fig. 5A and B; $\mathrm{P}<0.001)$. Furthermore, the results of the present study revealed that overexpression of miR-635 decreased TOP2A expression 
A

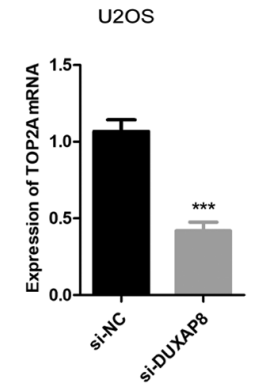

B
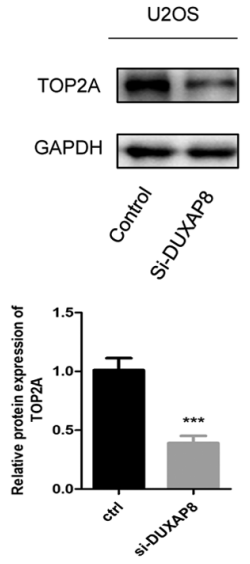

$\mathrm{E}$

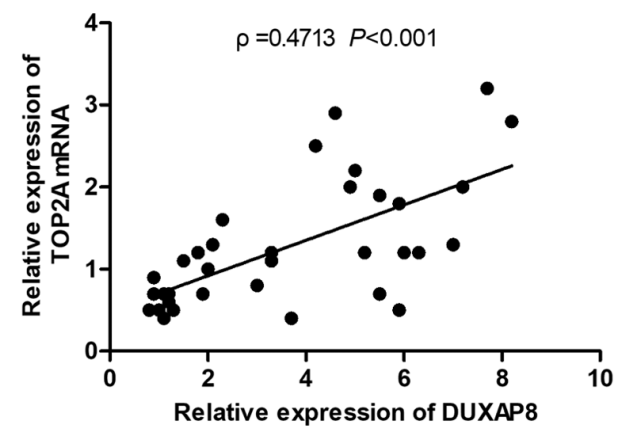

C
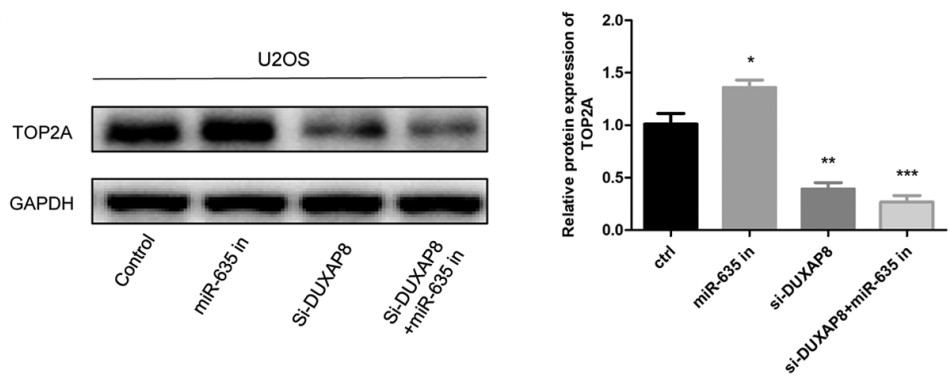

D
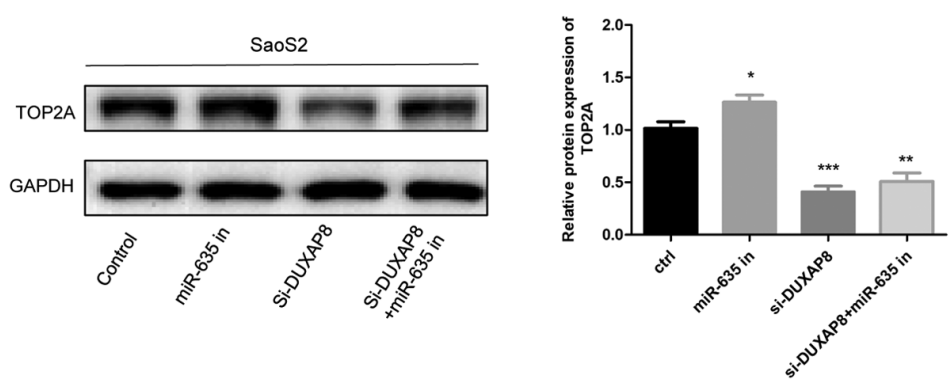

$\mathrm{F}$

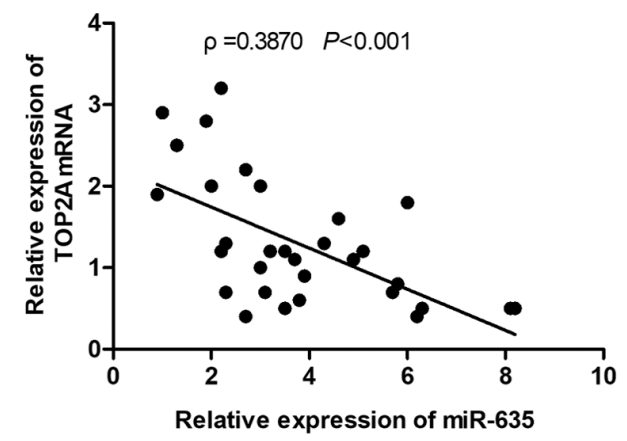

Figure 5. Regulation of DUXAP8/miR-635 on TOP2A. (A) Reverse transcription-quantitative polymerase chain reaction was used to detect the effect of DUXAP8-knockdown on TOP2A mRNA expression in OS cell lines, ${ }^{* * *} \mathrm{P}<0.001$ vs. control. (B) Western blotting was used to detect the effect of knockdown or overexpression of DUXAP8 on TOP2A protein expression in OS cell lines, ${ }^{* * * *} \mathrm{P}<0.001$ vs. control. (C and D) Western blotting was used to detect the effect of DUXAP8 and miR-635 on TOP2A protein expression in U2OS and SaoS2 cell lines, ${ }^{*} \mathrm{P}<0.05,{ }^{* * *} \mathrm{P}<0.01,{ }^{* * * *} \mathrm{P}<0.001$ vs. control. (E) The correlation between the expression levels of DUXAP8 and TOP2A mRNA in OS samples was analyzed. (F) The correlation between the expression levels of miR-635 and TOP2A mRNA in OS samples was analyzed. miR, microRNA; OS, osteosarcoma; siRNA, small interfering RNA; DUXAP8, double homeobox A pseudogene 8; TOP2A, topoisomerase alpha 2 ; NC, negative control.

and abolished the DUXAP8-induced upregulation of TOP2A (Fig. 5C and $\mathrm{D} ; \mathrm{P}<0.05$ ). Furthermore, it was found that the expression of TOP2A mRNA and DUXAP8 was positively correlated in OS tissue samples, while the expression of TOP2A mRNA was negatively correlated with the expression of miR-635 (Fig. 5E and F; $\mathrm{P}<0.001$ ). These results indicated that DUXAP8 may upregulate TOP2A expression in OS and promote OS progression, possibly by modulating miR-635.

\section{Discussion}

OS is thought to be one of most common causes of cancer-related mortality worldwide, with an 8\% 5-year survival rate (25). Increasing evidence has suggested that lncRNAs are involved in the development and progression of a diverse range of cancer types, including gastric cancer, hepatocellular carcinoma, clear cell renal cell carcinoma, colorectal cancer, breast cancer, non-small cell lung cancer and OS (26-29). For example, the IncRNA OSA3, which is specifically upregulated in prostate cancer, has been approved by the Food and Drug Administration for the diagnosis of prostate cancer (30). Downregulation of lncRNA HOST2 represses cell proliferation and promotes cell apoptosis in OS, which may offer a potential therapeutic target for OS (31). The regulation of IncRNA on OS proliferation and metastasis has been studied, but not for lncRNA DUXAP8. IncRNA DUXAP8 has been previously reported to be upregulated and may serve as a potential therapeutic target in several types of cancer (32). In a recent study, it was reported that lncRNA DUXAP8 enhances renal cell carcinoma progression by downregulating miR-126 (33). In the present study, it was found that IncRNA DUXAP8 was expressed at significantly higher levels in OS cell lines and tissues, suggesting that 
IncRNA DUXAP8 may contribute toward the progression of OS. Furthermore, the present study demonstrated that lncRNA DUXAP8-silencing significantly inhibited OS cell growth, cell migration and invasion ability, implying that lncRNA DUXAP8 serves an important role in OS progression.

MicroRNAs (miRNAs/miRs) are non-coding RNAs that serve an important regulatory role by acting as tumor promoters or suppressors in various cancer types. For example, miRNA-214 suppression contributes toward cell migration, invasion and EMT in gastric cancer by targeting FGFR (34). Previous studies have focused on the role of miRs in OS cells. However, few reports have demonstrated the effect of miR-635 in OS. A recent report demonstrated that miR-635 may accelerate the invasion of A375 melanoma cells (35). The present study demonstrated that miR-635 may function as a tumor suppressor in OS, as determined by experiments with human specimens and OS cell lines in an in vitro study. The present study demonstrated that miR-635 may be sponged by lncRNA DUXAP8 in OS. The binding relationship between DUXAP8 and miR-635 was validated using a dual-luciferase reporter gene assay. Additionally, knockdown of DUXAP8 significantly induced the expression of miR-635 in OS. Notably, with functional experiments, it was demonstrated that miR-635 could reverse the function of DUXAP8 in OS cells. These results not only explained the mechanism of miR-635 dysregulation in OS but also proved that miR-635 was a crucial effector during DUXAP8 regulation of the malignant phenotypes of OS cells. TOP2A has been previously reported to be upregulated in hepatocellular carcinoma $(36,37)$. The majority of studies have focused on TOP2A and reported the involvement of tumor chemoresistance to DNA-damaging agents in acute myeloid leukemia (AML) as well as other tumor types (38-40). The present study demonstrated that TOP2A may be involved in the development of OS cells as a carcinogenic factor and may be one of the candidate targets for miR-635. Knockdown of DUXAP8 significantly decreased TOP2A mRNA and protein expression, and DUXAP8 was positively correlated with TOP2A expression in OS tissues. Notably, the expression of miR-635 partially reversed the promotion of TOP2A expression caused by DUXAP8. These results demonstrated that DUXAP8 may target miR-635 to indirectly regulate TOP2A and thus affect the development of OS cells.

In conclusion, the results of the present study demonstrated that DUXAP8 is upregulated in OS tissues and cells. IncRNA DUXAP8-knockdown may suppress cell proliferation, cell migration and cell invasion in U2OS or SaOS2 cells. The present study also elucidated the mechanism of the DUXAP8/miR-635/TOP2A axis in the development of OS. With more in-depth research, DUXAP8 is likely to become a marker for clinical diagnosis and prognosis and potentially a therapeutic target for the treatment of OS.

\section{Acknowledgements}

Not applicable.

\section{Funding}

The present study was supported by the Science and Technology Research Project of the Jilin Provincial Department of Education (2020; grant no JJKH20200070KJ) and the Health Technology Innovation Project of Jilin Province (grant no. 2019J045).

\section{Availability of data and materials}

All data generated or analyzed during this study are included in this published article.

\section{Authors' contributions}

TY was responsible for conceiving the study. TY, JPG and FL curated the data. TY performed the formal analysis. TY and XLD undertook the investigations. TY and XLD were responsible for the methodology. HW, CX, JPG and FL performed the experiments. TY wrote the original draft. XLD reviewed and edited the manuscript. TY and XLD confirm the authenticity of all the raw data. All authors read and approved the final manuscript.

\section{Ethics approval and consent to participate}

All research protocols in the present study were approved by the Ethics Committee of the Affiliated Hospital of Bei Hua University. Written informed consent was obtained from every patient.

\section{Patient consent for publication}

Not applicable.

\section{Competing interests}

The authors declare that they have no competing interests.

\section{References}

1. Siegel RL, Miller KD and Jemal A: Cancer statistics, 2018. CA Cancer J Clin 68: 7-30, 2018.

2. Heare T,Hensley MA and Dell'Orfano S: Bone tumors: Osteosarcoma and Ewing's sarcoma. Curr Opin Pediatr 21: 365-372, 2009.

3. Harrison DJ, Geller DS, Gill JD, Lewis VO and Gorlick R: Current and future therapeutic approaches for osteosarcoma. Expert Rev Anticancer Ther 18: 39-50, 2018.

4. Strobel O, Neoptolemos J, Jäger D and Büchler MW: Optimizing the outcomes of osteosarcoma surgery. Nat Rev Clin Oncol 16: 11-26, 2019.

5. Isakoff MS, Bielack SS, Meltzer P and Gorlick R: Osteosarcoma: Current treatment and a collaborative pathway to success. J Clin Oncol 33: 3029-3035, 2015

6. Chen LL and Carmichael GG: Long noncoding RNAs in mammalian cells: What, where, and why? Wiley Interdiscip Rev RNA 1: 2-21, 2010

7. Iyer MK, Niknafs YS, Malik R, Singhal U, Sahu A, Hosono Y, Barrette TR, Prensner JR, Evans JR, Zhao S, et al: The landscape of long noncoding RNAs in the human transcriptome. Nat Genet 47: 199-208, 2015.

8. Prensner JR and Chinnaiyan AM: The emergence of lncRNAs in cancer biology. Cancer Discov 1: 391-407, 2011.

9. Rafiee A, Riazi-Rad F, Havaskary M and Nuri F: Long noncoding RNAs: Regulation, function and cancer. Biotechnol Genet Eng Rev 34: 153-180, 2018.

10. Mei D, Song H, Wang K, Lou Y, Sun W, Liu Z, Ding X and Guo J: Up-regulation of SUMO1 pseudogene 3 (SUMO1P3) in gastric cancer and its clinical association. Med Oncol 30: 709, 2013.

11. Lian Y, Xiong F, Yang L, Bo H, Gong Z, Wang Y, Wei F, Tang Y,LiX,LiaoQ, etal:Long noncoding RNA AFAP1-AS1 acts AS a competing endogenous RNA of miR-423-5p to facilitate nasopharyngeal carcinoma metastasis through regulating the $\mathrm{Rho} / \mathrm{Rac}$ pathway. J Exp Clin Cancer Res 37: 253, 2018. 
12. Xu LJ, Yu XJ, Wei B, Hui HX, Sun Y, Dai J and Chen XF: Long non-coding RNA DUXAP8 regulates proliferation and invasion of esophageal squamous cell cancer. Eur Rev Med Pharmacol Sci 22: 2646-2652, 2018.

13. Ma HW, Xie M, Sun M, Chen TY, Jin RR, Ma TS, Chen QN, Zhang EB, He XZ, De W and Zhang ZH: The pseudogene derived long noncoding RNA DUXAP8 promotes gastric cancer cell proliferation and migration via epigenetically silencing PLEKHO1 expression. Oncotarget 8: 52211-52224, 2016.

14. Jiang $\mathrm{H}$, Shi $\mathrm{X}, \mathrm{Ye} \mathrm{G}, \mathrm{Xu} \mathrm{Y}, \mathrm{Xu} \mathrm{J}, \mathrm{Lu} \mathrm{J}$ and $\mathrm{Lu} \mathrm{W}$ : Up-regulated long non-coding RNA DUXAP8 promotes cell growth through repressing Krüppel-like factor 2 expression in human hepatocellular carcinoma. Onco Targets Ther 11: 7429-7436, 2019.

15. AmbRoS V: The functions of animal microRNAs. Nature 431: 350-355, 2004.

16. Cummins JM, He Y, Leary RJ, Pagliarini R, Diaz LA Jr, Sjoblom T, Barad O, Bentwich Z, Szafranska AE, Labourier E, et al: The colorectal microRNAome. Proc Nat Acad Sci USA 103: 3687-3692, 2006.

17. Weber CE, Luo C, Hotz-Wagenblatt A, Gardyan A, Kordass T, Holland-Letz T, Osen W and Eichmüller SB: miR-339-3p is a tumor suppressor in melanoma. Cancer Res 76: 3562-3571, 2016.

18. Jain M, Zhang L, He M, Zhang YQ, Shen M and Kebebew E: TOP $2 \mathrm{~A}$ is overexpressed and is a therapeutic target for adrenocortical carcinoma. Endocr Relat Cancer 20: 361-370, 2013.

19. Ejlertsen B, Jensen MB, Nielsen KV, Balslev E, Rasmussen BB Willemoe GL, Hertel PB, Knoop AS, Mouridsen HT and Brünner N: HER2, TOP2A, and TIMP-1 and responsiveness to adjuvant anthracycline-containing chemotherapy in high-risk breast cancer patients. J Clin Oncol 28: 984-990, 2010.

20. Zhang S, Jiang H, Xu Z, Jiang Y, She Y, Huang X, Feng S, Chen W, Chen $\mathrm{S}$, Chen $\mathrm{Y}$, et al: The resistance of esophageal cancer cells to paclitaxel can be reduced by the knockdown of long noncoding RNA DDX11-AS1 through TAF1/TOP2A inhibition. Am J Cancer Res 9: 2233-2248, 2019.

21. Livak KJ and Schmittgen TD: Analysis of relative gene expression data using real-time quantitative PCR and the 2(-Delta Delta C(T)) method. Methods 25: 402-408, 2001.

22. $\mathrm{Yu} \mathrm{H}, \mathrm{Xu} \mathrm{Y}$, Zhang D and Liu G: Long noncoding RNA LUCAT1 promotes malignancy of ovarian cancer through regulation of miR-612/HOXA13 pathway. Biochem Biophys Res Commun 503: 2095-2100, 2018.

23. Rojo AI, Medina-Campos ON, Rada P, Zúñiga-Toala A, López-Gazcón A, Espada S, Pedraza-Chaverri J and Cuadrado A: Signaling pathways activated by the phytochemical nordihydroguaiaretic acid contribute to a Keapl-independent regulation of Nrf2 stability: Role of glycogen synthase kinase-3. Free Radic Biol Med 52: 473-487, 2012.

24. Golabchi K, Soleimani-Jelodar R, Aghadoost N, Momeni F, Moridikia A, Nahand JS, Masoudifar A, Razmjoo H and Mirzaei H: MicroRNAs in retinoblastoma: Potential diagnostic and therapeutic biomarkers. J Cell Physiol 233: 3016-3023, 2018.

25. Lulla RR, Costa FF, Bischof JM, Chou PM, de F Bonaldo M, Vanin EF and Soares MB: Identification of differentially expressed microRNAs in osteosarcoma. Sarcoma 2011: 732690 2011.

26. Yang C, Wu K, Wang S and Wei G: Long non-coding RNA XIST promotes osteosarcoma progression by targeting YAP via miR-195-5p. J Cell Biochem 119: 5646-5656, 2018.
27. Liu X, Wang Y, Sun L, Min J, Liu J, Chen D, Zhang H, Zhang $\mathrm{H}$, Zhang $\mathrm{H}$, Zhou $\mathrm{Y}$ and Liu L: Long noncoding RNA BC005927 upregulates EPHB4 and promotes gastric cancer metastasis under hypoxia. Cancer Sci 109: 988-1000, 2018.

28. Liang Y, Ma Y, Li L, Shen X, Xin T, Zhao Y and Ma R Effect of long non-coding RNA LINC01116 on biological behaviors of non-small cell lung cancer cells via the hippo signaling pathway. J Cell Biochem 119: 6310, 2018.

29. Zhang Y, Tao Y, Li Y, Zhao J, Zhang L, Zhang X, Dong C, Xie Y, Dai X, Zhang X and Liao Q: The regulatory network analysis of long noncoding RNAs in human colorectal cancer. Funct Integr Genomics 18: 261-275, 2018.

30. An N and Cheng D: The Long Noncoding RNA HOST2 promotes gemcitabine resistance in human pancreatic cancer cells. Pathol Oncol Res 26: 425-431, 2020.

31. Lu W, Zhang H, Niu Y, Wu Y, Sun W, Li H, Kong J, Ding K, Shen HM, Wu H, et al: Long non-coding RNA lincDUXAP8 regulated non-small cell lung cancer proliferation, migration, invasion and epithelial mesenchymal transition by sponging miR-150-5p. Mol Cancer 16: 118, 2017.

32. Lin MG, Hong YK, Zhang Y, Lin BB and He XJ: Mechanism of lncRNA DUXAP8 in promoting proliferation of bladder cancer cells by regulating PTEN. Eur Rev Med Pharmacol Sci 22: 3370-3377, 2018

33. Huang T, Wang X, Yang X, Ji J, Wang Q, Yue X and Dong Z: Long non-coding RNA DUXAP8 enhances renal cell carcinoma progression via downregulating miR-126. Med Sci Monit 14: 7340-7347, 2018

34. Wang R, Sun Y, Yu W, Yan Y, Qiao M, Jiang R, Guan W and Wang L: Downregulation of miRNA-214 in cancer-associated fibroblasts contributes to migration and invasion of gastric cancer cells through targeting FGF9 and inducing EMT. J Exp Clin Cancer Res 38: 20, 2019.

35. Weber CE, Luo C, Hotz-Wagenblatt A, Gardyan A, Kordass T, Holland-Letz T, Osen W and Eichmüller SB: miR-339-3p is a tumor suppressor in melanoma. Cancer Res 76: 3562-3571, 2019.

36. Wong N, Yeo W, Wong WL, Wong NL, Chan KY, Mo FK, Koh J, Chan SL, Chan AT, Lai PB, et al: TOP2A overexpression in hepatocellular carcinoma correlates with early age onset, shorter patients' survival and chemoresistance. Int J Cancer 124: 644-652, 2009.

37. Panvichian R, Tantiwetrueangdet A, Angkathunyakul N and Leelaudomlipi S: TOP2A amplification and overexpression in hepatocellular carcinoma tissues. Biomed Res Int 2015: 381602, 2015.

38. Chekerov R, Klaman I, Zafrakas M, Könsgen D, Mustea A, Petschke B, Lichtenegger W, Sehouli J and Dahl E: Altered expression pattern of topoisomerase IIalpha in ovarian tumor epithelial and stromal cells after platinum-based chemotherapy. Neoplasia 8: 38-45, 2006

39. Chen CC, Gau JP, You JY, Lee KD, Yu YB, Lu CH, Lin JT, Lan C, Lo WH, Liu JM and Yang CF: Prognostic significance of beta-catenin and topoisomerase IIalpha in de novo acute myeloid leukemia. Am J Hematol 84: 87-92, 2009.

40. Chen CF, He X, Arslan AD, Mo YY, Reinhold WC, Pommier Y and Beck WT: Novel regulation of nuclear factor-YB by miR-485-3p affects the expression of DNA topoisomerase IIa and drug responsiveness. Mol Pharmacol 79: 735-741, 2011.

This work is licensed under a Creative Commons Attribution-NonCommercial-NoDerivatives 4.0 International (CC BY-NC-ND 4.0) License. 